\title{
On an Algorithm for Dynamic Reconstruction in Systems with Delay in Control
}

\author{
Marina Blizorukova* \\ Ural Federal University and Institute of Mathematics and Mechanics, \\ Ural Branch of the Russian Academy of Sciences, \\ Ekaterinburg, 620990 Russia \\ msb@imm.uran.ru
}

\begin{abstract}
We discuss a problem of the dynamic reconstruction of unknown input controls in nonlinear vector equations. A regularizing algorithm is proposed for reconstructing these controls simultaneously with the processes. The algorithm is stable with respect to informational noises and computational errors.
\end{abstract}

Keywords: dynamic reconstruction, method of auxiliary models.

\section{Introduction Problem Statement}

Consider a controlled system described by the following equation

$$
\dot{x}(t)=f_{1}\left(t, u_{t}(s), x_{t}(s)\right)+f_{2}\left(t, x_{t}(s)\right) u(t)
$$

with the initial state

$$
u_{t_{0}}(s)=u_{0}(s) \in C\left(\left[-\tau_{m}^{u}, 0\right] ; R^{n_{1}}\right), \quad x_{t_{0}}(s)=x_{0}(s) \in C\left(\left[-\tau_{n}^{x}, 0\right] ; R^{n_{2}}\right) .
$$

Here $t$ is time from a fixed interval $T=\left[t_{0}, \vartheta\right]\left(t_{0}<\vartheta<+\infty\right) ; x(t)=$ $\left(x_{1}(t), \ldots, x_{n_{2}}(t)\right)$ is the phase state of the system; $u(t)=\left(u_{1}(t), \ldots, u_{n_{1}}(t)\right)$ is a control; the symbols $x_{t}(s)$ and $u_{t}(s)$ mean the functions $x_{t}(s)=x(t+s)$ for $s \in\left[-\tau_{n}^{x}, 0\right]$ and $u_{t}(s)=u(t+s)$ for $s \in\left[-\tau_{m}^{u}, 0\right]$, respectively. We assume that initial state (2) is Lipschitz. For simplicity, we assume also that the initial state $x_{0}(s), u_{0}(s)$ is fixed and known. The control $u=u(t)=\left(u_{1}(t), \ldots, u_{n_{1}}(t)\right)$ is called an admissible control if its components $u_{i}(t), i \in\left[1: n_{1}\right]$, are Lebesgue measurable functions on the interval $T$ and values $u(t)$ belong to a given compact set $P$ from Euclidean space $R^{n_{1}}$ for almost all $t \in T$. The set of all admissible controls is denoted by $P(\cdot)$. Therefore, $P(\cdot)=\left\{u(\cdot) \in L_{2}\left(T ; R^{n_{1}}\right): u(t) \in P\right.$ for a. a. $t \in T\}$. By the trajectory (or the solution) $x(\cdot)$ of equation (11) with initial state (2) corresponding to some admissible control $u(\cdot)$, we call absolutely continuous on $T$ function $x=x(t)$ satisfying (1) for a.a. $t \in T$.

\footnotetext{
* This work was supported by the Russian Foundation for Basic Research (12-0100175-a), by the Ural-Siberian Integration Project (12-C-1-1017), and by the Program for support of leading scientific schools of Russia (6512.2012.1).
}

D. Hömberg and F. Tröltzsch (Eds.): CSMO 2011, IFIP AICT 391, pp. 225-234, 2013.

(C) IFIP International Federation for Information Processing 2013 
Condition 1. The elements of matrix function

$f_{2 i j}\left(t, x_{t}(s)\right)=f_{2 i j}\left(t, x(t), x\left(t-\tau_{1}^{x}\right), \ldots, x\left(t-\tau_{n}^{x}\right)\right), \quad i \in\left[1: n_{2}\right], \quad j \in\left[1: n_{1}\right]$,

and vector-valued function

$$
\begin{gathered}
f_{1 i}\left(t, u_{t}(s), x_{t}(s)\right)= \\
=f_{1 i}\left(t, u\left(t-\tau_{1}^{u}\right), \ldots, u\left(t-\tau_{m}^{u}\right), x(t), x\left(t-\tau_{1}^{x}\right), \ldots, x\left(t-\tau_{n}^{x}\right)\right), \quad i \in\left[1: n_{2}\right]
\end{gathered}
$$

satisfy the Lipschitz conditions

$$
\begin{gathered}
\left|f_{2 i j}\left(t_{1}, x_{0}^{(1)}, x_{1}^{(1)}, \ldots, x_{n}^{(1)}\right)-f_{2 i j}\left(t_{2}, x_{0}^{(1)}, x_{1}^{(2)}, \ldots, x_{n}^{(2)}\right)\right| \leq \\
\leq C_{1}\left(\left|t_{2}-t_{1}\right|+\sum_{j=0}^{n}\left|x_{j}^{(1)}-x_{j}^{(2)}\right|\right) \\
\left|f_{1 i}\left(t_{1}, u_{1}^{(1)}, \ldots, u_{m}^{(1)}, x_{0}^{(1)}, x_{1}^{(1)}, \ldots, x_{n}^{(1)}\right)-f_{1 i}\left(t_{2}, u_{1}^{(2)}, \ldots, u_{m}^{(2)}, x_{0}^{(2)}, x_{1}^{(2)}, \ldots, x_{n}^{(2)}\right)\right| \\
\leq d_{1}\left(\left|t_{2}-t_{1}\right|+\sum_{i=1}^{m}\left|u_{i}^{(1)}-u_{i}^{(2)}\right|+\sum_{j=0}^{n}\left|x_{j}^{(1)}-x_{j}^{(2)}\right|\right)
\end{gathered}
$$

In this case, under this condition for any pair, i.e., for initial state (2) and the control $u(\cdot) \in P(\cdot)$, there exists a unique solution of equation (11).

Let $u(\cdot)$ be an admissible control realizing during the given time interval $T$; $x(\cdot)$ be the real motion generated by this control. We assume that the phase states $x\left(\tau_{i}\right)$ of the system are inaccurately measured at frequent enough time moments $\tau_{i} \in T$ in the process. Measurement results $\xi^{h}\left(\tau_{i}\right) \in R^{n_{2}}$ satisfy the inequalities

$$
\left|\xi^{h}\left(\tau_{i}\right)-x\left(\tau_{i}\right)\right| \leq h
$$

Here, the quantity $h \in(0,1)$ specifies the measurement error.

In the present paper, we construct an algorithm that reconstructs the control $u(\cdot)$ on the basis of the current information $\xi^{h}(\cdot)$ in real time. Since the exact reconstruction is impossible due to the error of measurements $\xi^{h}(\cdot)$ we require that the algorithm should generate some approximation. Namely, it is required to construct an algorithm allowing us, on the basis of the inaccurate measurements $\xi^{h}(\cdot)$, and in real time, to form the admissible control $v^{h}(\cdot)$ such that the meansquare deviation of $v^{h}(\cdot)$ from $u(\cdot)$ :, i.e.,

$$
\left|v^{h}(\cdot)-u(\cdot)\right|_{L_{2}(T)}^{2}=\int_{t_{0}}^{\vartheta}\left|v^{h}(t)-u(t)\right|^{2} d t,
$$

is arbitrarily small for the sufficiently small measurement error $h$. Since the measurements are inaccurate it is in general impossible to identify $u(t)$ precisely, therefore the problem is to approximate the input by some function $v^{h}(t)$. 
Here and below, the symbol $|\cdot|$ stands for both the Euclidean norm and the corresponding matrix norm and for the modulo of a number. In what follows, we set $\tau_{m}^{u}=\tau_{n}^{x}=\tau$ for simplicity, and by $\xi^{h}(\cdot)$ we denote the function $\xi^{h}(t)$, $t \in\left[t_{0}-\tau, \vartheta\right]$ such that $\xi^{h}(t)=x_{0}\left(t-t_{0}\right)$ for $t \in\left[t_{0}-\tau, t_{0}\right), \xi^{h}(t)=\xi^{h}\left(\tau_{i}\right)$ for $t \in\left[\tau_{i}, \tau_{i+1}\right), i \in[0: d-1]$, where $\tau_{i}=\tau_{h, i}, d=d_{h}, \xi^{h}\left(\tau_{i}\right)$ satisfies (5).

The suggested solution outline is the following ( [1 6$]$ ). An auxiliary control system (model $M$ ) described by equation of the form

$$
\dot{w}(t)=F\left(t, \xi_{t}^{h}(s), v_{t}^{h}(s)\right), \quad w_{t_{0}}(s)=w_{0}(s), \quad t \in T
$$

is associated with the real dynamical system (10). Here the vector $w \in R^{n_{2}}$ characterizes state of the model, the form of function $F$ is corrected below, vector $v^{h}$ is control action. After that, the problem of reconstruction of input $u(\cdot)$ is replaced by the problem of positional control of the model. This process is realized on the time interval $T$ in such a way that control $v^{h}(\cdot)$ "approximates" appropriately $u(\cdot)$. First, one takes a uniform net $\Delta=\left\{\tau_{i}\right\}_{i=0}^{m}, \tau_{i+1}=\tau_{i}+\delta$, $\delta>0, i \in[0: m], \tau_{0}=0, \tau_{m}=T$ with the step $\delta$. Then, on the interval $t \in\left[\tau_{i}, \tau_{i+1}\right)$ the model is acted upon the controls

$$
v_{i}^{h}=V_{h}\left(\tau_{i}, w_{\tau_{i}}(s), \xi_{\tau_{i}}^{h}(s)\right)
$$

calculated at the moment $\tau_{i}$ by use of some rule, which hereinafter we shall identify with mapping $V_{h}$. Thus, the controls in the model are realized by the method of feedback control. Its value on the interval $\left[\tau_{i}, \tau_{i+1}\right]$ depends on the measurement results $\xi^{h}(\cdot)$ corresponding to the phase state $x(\cdot)$ of the system (1) and state $w$ of the model (7). The described process forms the piece-wise function

$$
v^{h}(t)=v_{i}^{h}, \quad t \in\left[\tau_{i}, \tau_{i+1}\right)
$$

in real time synchro with the motion of real system (1). Thus, to solve the problem above, we should specify a model and a control law for this model.

\section{Algorithm for Solving the Problem}

As a model, we take the following system of linear ordinary differential equation

$$
\begin{gathered}
\dot{w}(t)=f_{1}\left(\tau_{i}, v_{\tau_{i}}^{h}(s), \xi_{\tau_{i}}^{h}(s)\right)+f_{2}\left(\tau_{i}, \xi_{\tau_{i}}^{h}(s)\right) v_{i}^{h}+2\left(\xi^{h}\left(\tau_{i}\right)-w\left(\tau_{i}\right)\right), \\
w \in R^{n_{2}}, \quad t \in\left[\tau_{i}, \tau_{i+1}\right), \quad \tau_{i}=\tau_{h, i}, \quad v_{t_{0}}^{h}(s)=u_{0}(s),
\end{gathered}
$$

with the initial state $w\left(t_{0}\right)=\xi^{h}\left(t_{0}\right)$. The solution of this equation $w(\cdot)=$ $w\left(\cdot ; t_{0}, w_{t_{0}}(s), v^{h}(\cdot)\right)$ is understood in the sense of Caratheodory. So, the righthand side of equation of the model (7) has the form

$$
\begin{gathered}
F\left(t, \xi_{t}^{h}(s), v_{t}^{h}(s)\right)=f_{1}\left(\tau_{i}, v_{\tau_{i}}^{h}(s), \xi_{\tau_{i}}^{h}(s)\right)+f_{2}\left(\tau_{i}, \xi_{\tau_{i}}^{h}(s)\right) v_{i}^{h}+ \\
+2\left(\xi^{h}\left(\tau_{i}\right)-w\left(\tau_{i}\right)\right), \quad t \in\left[\tau_{i}, \tau_{i+1}\right) .
\end{gathered}
$$


Introduce the following notation: $\Delta^{(j)}=\left[t_{j}, t_{j+1}\right], \quad t_{j}=t_{0}+\tau_{1}^{x} j$; the symbol $l$ stands for the integer part of the number $\tau / \tau_{1}^{x} ; j_{*}=\max \left\{j: t_{j}<\vartheta\right\}$,

$$
g_{j}(h)=h^{(1 / 3)^{j}}, \quad j \in\left[1: j_{*}\right] .
$$

Fix a partition of the interval $T$ with a step $\delta=\delta(h)$ depending on the measurement error $h$, i.e.,

$$
\Delta_{h}=\left\{\tau_{h, i}\right\}_{i=0}^{d_{h}}, \quad \tau_{i}=\tau_{h, i}, \quad \tau_{h, 0}=t_{0}, \quad \tau_{h, d_{h}}=\vartheta
$$

(for simplicity, we assume that $\tau_{i}-\tau_{i-1}=\delta=\delta(h)$ ). Without loss of generality, we can suppose that the partition $\Delta_{h}$ is chosen in such a way that $t_{j} \in \Delta_{h}$. Define the law of forming the control $v_{i}^{h}$ in the model (for $\tau_{i} \in\left[t_{j}, t_{j+1}\right) \cap T$ ) by the relations

$$
\begin{aligned}
& V_{h}\left(\tau_{i}, w_{\tau_{i}}(s), \xi_{\tau_{i}}^{h}(s)\right)=V_{j}\left(\tau_{i}, w_{\tau_{i}}(s), \xi_{\tau_{i}}^{h}(s)\right) \\
= & \arg \min \left\{2\left(l_{i}, f_{2}\left(\tau_{i}, \xi_{\tau_{i}}^{h}(s)\right) v\right)+\alpha_{j}|v|^{2}: v \in P\right\} .
\end{aligned}
$$

Here $\alpha_{j}$ is a parameter, $j \in\left[0: j_{*}\right], l_{i}=w\left(\tau_{i}\right)-\xi^{h}\left(\tau_{i}\right)$.

Condition 2. Let $n_{2} \geq n_{1}$, and let there exists a number $c_{*}>0$ such that the matrix $f_{2}\left(t, x_{t}(s)\right)$ has a minor of order $n_{1}$ with the property: the $n_{1} \times n_{1}$ dimensional matrix $\bar{f}_{2}(t)=\bar{f}_{2}\left(t, x_{t}(s)\right)$ corresponding to this minor satisfies the inequality

$$
\left|\bar{f}_{2}(t) u\right| \geq c_{*}|u|
$$

for each $t \in T$ and all $u \in R^{n_{1}}$.

We choose the parameter $\alpha_{j}$ which plays the role of the regularizer, as follows:

$$
\alpha_{0}=C h^{2 / 3}, \quad \alpha_{j}=C g_{j}^{2 / 3}(h), \quad j \geq 1, \quad C=\text { const }>0 .
$$

Let us describe the algorithm for solving the problem above.

Before the initial moment the value $h$ and the partition $\Delta=\Delta_{h}$ with diameter $\delta=\delta(h)$ are fixed. The work of the algorithm starting at time $t=0$ is decomposed into $m_{h}-1$ steps. At the $i$-th step carried out during the time interval $\delta_{i}=\left[\tau_{i}, \tau_{i+1}\right), \tau_{i}=\tau_{h, i}$, the following actions take place. First, at time moment $\tau_{i}$ vector $v_{i}^{h}$ is calculated by formula (11). Then the control $v^{h}(t)=v_{i}^{h}$ is fed onto the input of the model (9). After that, we transform the state $w_{\tau_{i}}(s)$ of the model into $w_{\tau_{i+1}}(s)$. The procedure stops at time $\vartheta$.

The following theorem is true.

Theorem 1. Let $\delta=\delta(h) \leq h$. Then the inequalities

$$
\nu^{(j)} \equiv\left|v^{h}(\cdot)-u(\cdot)\right|_{L_{2}\left(\Delta^{(j-1)} ; R^{n_{1}}\right)}^{2} \leq c_{j} g_{j}(h), \quad j \in\left[1: j_{*}\right],
$$

are valid. Here, $v^{h}(t)=u(t)$ for $t \in\left[t_{0}-\tau, t_{0}\right], v^{h}(t)=u_{0}(-\tau)$ for $t \in\left[t_{0}-\tau-\right.$ $\left.\tau_{1}^{u}, t_{0}-\tau\right)$. 
The proof of the theorem is based on auxiliary statements, which are used in forthcoming considerations. Introduce two systems

$$
\begin{aligned}
& \dot{p}(t)=f_{1}(t)+f_{2}(t) u_{1}(t), \quad t \in T, \\
& \dot{q}(t)=F_{1}(t)+F_{2}(t) u_{2}(t),
\end{aligned}
$$

where $p(t), q(t) \in R^{n}, f_{1}(\cdot), F_{1}(\cdot) \in L_{2}\left(T ; R^{n}\right), f_{2}(\cdot) \in L_{2}\left(T ; R^{n \times r}\right), F_{2}(\cdot) \in$ $L_{2}\left(T ; R^{n \times r}\right), u_{1}(\cdot), u_{2}(\cdot) \in L_{2}\left(T ; R^{r}\right),\left|u_{l}(\cdot)\right|_{L_{\infty}\left(T ; R^{r}\right)} \leq K, l=1,2$.

Introduce the notation: $\Delta_{*}^{(j)}=\left[t_{j}^{*}, t_{j+1}^{*}\right] \cap T, t_{j}^{*}=t_{0}+\tau_{*} j, j \in\left[\begin{array}{lll}0: j_{0}\end{array}\right]$, $\Delta^{(-1)}=\left[t_{0}-\tau_{*}, t_{0}\right], \tau_{*}=$ const $\in\left(0, \vartheta-t_{0}\right), j_{0}=\max \left\{j: t_{j}^{*} \leq \vartheta\right\}$. Let $r \leq n$ and let there exists a number $c>0$ such that the matrix $f_{2}(t)$ has a minor of order $r$ such that the $r \times r$-matrix $\bar{f}_{2}(t)$ corresponding to this minor satisfies the following inequality: $\left|\bar{f}_{2}(t) u\right| \geq c|u|$ for each $t \in T$ and all $u \in R^{r}$.

It is easy to verify the following lemmas.

Lemma 1. Let the function $t \rightarrow\left(\bar{f}_{2}(t)\right)^{-1} u_{1}(t)$ be a function of bounded variation on $T$ and let the conditions

$$
\begin{gathered}
\left|f_{1}(\cdot)-F_{1}(\cdot)\right|_{L_{2}\left(\Delta_{*}^{(j)} ; R^{n}\right)}^{2} \leq a_{1}^{(j)}, \quad\left|f_{2}(\cdot)-F_{2}(\cdot)\right|_{L_{2}\left(\Delta_{*}^{(j)} ; R^{n \times r}\right)}^{2} \leq a_{2}^{(j)}, \\
|p(t)-q(t)|^{2}+\tilde{\alpha}_{j} \int_{t_{j}^{*}}^{t}\left\{\left|u_{2}(\nu)\right|^{2}-\left|u_{1}(\nu)\right|^{2}\right\} d \nu \leq a_{3}^{(j)} \quad t \in\left[t_{j}^{*}, t_{j+1}^{*}\right], \\
\left|p\left(t_{j}^{*}\right)-q\left(t_{j}^{*}\right)\right|^{2} \leq a_{4}^{(j)}, \quad \tilde{\alpha}_{j}=\text { const } \in(0,+\infty)
\end{gathered}
$$

be true. Then the inequality

$$
\left|u_{1}(\cdot)-u_{2}(\cdot)\right|_{L_{2}\left(\Delta_{*}^{(j)} ; R^{r}\right)}^{2} \leq K_{j}\left\{\sum_{l=1}^{4}\left(a_{l}^{(j)}\right)^{1 / 2}+\tilde{\alpha}_{j}^{1 / 2}\right\}+a_{3}^{(j)} / \tilde{\alpha}_{j}
$$

is valid.

Lemma 2. The bunches of solutions of systems (1) and (9) are bounded in the space $W^{1, \infty}\left(T ; R^{n_{2}}\right)=\left\{x(\cdot) \in L_{2}\left(T ; R^{n_{2}}\right) ; \dot{x}(\cdot) \in L_{2}\left(T ; R^{n_{2}}\right)\right\}$.

We use the relation

$$
\varepsilon_{j}(t)=|x(t)-w(t)|^{2}+\alpha_{j} \int_{t_{j}}^{t}\left\{\left|v^{h}(\nu)\right|^{2}-|u(\nu)|^{2}\right\} d \nu, \quad j \in\left[0: j_{*}\right], \quad t \in T .
$$

Lemma 3. The following inequalities

$$
\varepsilon_{j}(t) \leq b_{j}, \quad t \in \Delta^{(j)} \cap T, \quad j \in\left[0: j_{*}\right],
$$

are valid, where

$$
b_{j}=\left|x\left(t_{j}\right)-w\left(t_{j}\right)\right|^{2}+c_{j}^{(1)}(h+\delta)+c_{j}^{(2)} \sum_{k=j-l}^{j} \nu^{(k)},
$$

$c_{j}^{(1)}, c_{j}^{(2)}$ are some constants, which can be explicitly written. 
Proof. Fix $\tau_{i} \in \Delta^{(j)}$. Then for $t \in \Delta^{(j)} \cap \delta_{i}=\left[\tau_{i}, \tau_{i+1}\right]$, we obtain

$$
\varepsilon_{j}(t) \leq \varepsilon_{j}\left(\tau_{i}\right)+\sum_{j=1}^{4} \Lambda_{j i}(t),
$$

where

$$
\begin{gathered}
\Lambda_{1 i}(t)=2\left(s_{i}, \int_{\tau_{i}}^{t}\left\{f_{1}\left(\nu, u_{\nu}(s), x_{\nu}(s)\right)-f_{1}\left(\tau_{i}, v_{\nu}^{h}(s), \xi_{\tau_{i}}^{h}(s)\right)\right\} d \nu\right), s_{i}=x\left(\tau_{i}\right)-w\left(\tau_{i}\right), \\
\Lambda_{2 i}(t)=2\left(s_{i}, \int_{\tau_{i}}^{t}\left\{f_{2}\left(\nu, x_{\nu}(s)\right) u(\nu)-\right.\right. \\
\left.\left.-f_{2}\left(\tau_{i}, \xi_{\tau_{i}}^{h}(s)\right) v_{i}^{h}\right\} d \nu\right)+\alpha_{j} \int_{\tau_{i}}^{t}\left\{\left|v^{h}(\nu)\right|^{2}-|u(\nu)|^{2}\right\} d \tau, \\
\Lambda_{3 i}(t)=-2\left(t-\tau_{i}\right)\left(s_{i}, \xi^{h}\left(\tau_{i}\right)-w\left(\tau_{i}\right)\right), \quad \Lambda_{4 i}(t)=\left(t-\tau_{i}\right) \int_{\tau_{i}}^{t}|\dot{w}(\tau)-\dot{x}(\tau)|^{2} d \tau .
\end{gathered}
$$

By virtue of lemma 2, we have

$$
\Lambda_{4 i}(t) \leq K_{*}^{(j)}\left(t-\tau_{i}\right)^{2}, \quad t \in \delta_{i} .
$$

Note that $v^{h}\left(\tau_{i}+s\right)=v^{h}(t+s)$ for $s \geq t_{0}-\tau_{i}, t \in\left[\tau_{i}, \tau_{i+1}\right]$ and in addition

$$
\left|\xi^{h}\left(\tau_{i}+s\right)-x(t+s)\right| \leq K_{*}\left(h+t-\tau_{i}\right) \quad \text { for } \quad \tau_{i}+s \geq t_{0}-\tau .
$$

Taking into account lemma 2, as well as the Lipschitz property of the functions $u_{0}(s)$ and $x_{0}(s)$, inequalities (4) and the relation

$$
\left|\xi^{h}\left(\tau_{i}+s\right)-x(t+s)\right| \leq K_{*}\left(h+t-\tau_{i}\right) \quad \text { for } \quad \tau_{i}+s \geq t_{0}-\tau,
$$

we obtain for $t \in \delta_{i}$ the estimate

$$
\begin{gathered}
\int_{\tau_{i}}^{t}\left|f_{1}\left(\nu, u_{\nu}(s), x_{\nu}(s)\right)-f_{1}\left(\tau_{i}, v_{\nu}^{h}(s), \xi_{\tau_{i}}^{h}(s)\right)\right| d \nu \leq \\
\leq K_{1}^{(j)}\left(t-\tau_{i}\right)\left(h+t-\tau_{i}\right)+K_{2}^{(j)}\left(t-\tau_{i}\right)^{1 / 2} \sum_{k=1}^{m}\left(\int_{\tau_{i}-\tau_{k}^{u}}^{t-\tau_{k}^{u}}\left|u(\nu)-v^{h}(\nu)\right|^{2} d \nu\right)^{1 / 2} .
\end{gathered}
$$

Here, $\tau_{0}^{x}=0$. In this case, the inequality

$$
\Lambda_{1 i}(t) \leq 2\left(t-\tau_{i}\right)\left|x\left(\tau_{i}\right)-w\left(\tau_{i}\right)\right|^{2}+K_{3}^{(j)}\left\{\left(t-\tau_{i}\right)\left(h+t-\tau_{i}\right)^{2}+\right.
$$




$$
\left.+\sum_{k=1}^{m} \int_{\tau_{i}-\tau_{k}^{u}}^{t-\tau_{k}^{u}}\left|u(\nu)-v^{h}(\nu)\right|^{2} d \nu\right\}
$$

holds for $t \in \delta_{i}$. In view of (므), we have

$$
\Lambda_{3 i}(t) \leq-2\left(t-\tau_{i}\right)\left|x\left(\tau_{i}\right)-w\left(\tau_{i}\right)\right|^{2}+K_{4}^{(j)} h\left(t-\tau_{i}\right), \quad t \in \delta_{i} .
$$

Moreover, from (5), (3), and (16), we derive

$$
\left|f_{2}\left(\nu, x_{\nu}(s)\right) u(\nu)-f_{2}\left(\tau_{i}, \xi_{\tau_{i}}^{h}(s)\right) u(\nu)\right| \leq K_{0}\left(h+\nu-\tau_{i}\right)
$$

for $\nu \in\left[\tau_{i}, \tau_{i+1}\right]$. In this case,

$$
\begin{gathered}
\Lambda_{2 i}(t) \leq K_{5}^{(j)}\left(t-\tau_{i}\right)\left(h+t-\tau_{i}\right)+ \\
+\int_{\tau_{i}}^{t}\left\{2\left(l_{i}, f_{2}\left(\tau_{i}, \xi_{\tau_{i}}^{h}(s)\right)\left\{v_{i}^{h}-u(\nu)\right\}+\alpha_{j}\left\{\left|v_{i}^{h}\right|^{2}-|u(\nu)|^{2}\right\}\right\} d \nu .\right.
\end{gathered}
$$

The rule for forming the control $v_{i}^{h}$ (11) and the last inequality imply

$$
\Lambda_{2 i}(t) \leq K_{5}^{(j)}\left(t-\tau_{i}\right)\left(h+t-\tau_{i}\right)
$$

Finally, taking into account (13) $-(19)$, we conclude that for $t \in \Delta^{(j)} \cap \delta_{i}$

$$
\varepsilon_{j}(t) \leq \varepsilon_{j}\left(\tau_{i}\right)+K_{6}^{(j)} \delta(h+\delta)+K_{3}^{(j)} \sum_{k=1}^{m} \int_{\tau_{i}-\tau_{k}^{u}}^{t-\tau_{k}^{u}}\left|u(\nu)-v^{h}(\nu)\right|^{2} d \nu,
$$

i.e., for $t \in \Delta^{(j)}=\left[t_{j}, t_{j+1}\right]$,

$$
\varepsilon_{j}(t) \leq \varepsilon_{j}\left(t_{j}\right)+K_{7}^{(j)}(h+\delta)+K_{8}^{(j)} \int_{t_{j}-\tau}^{t_{j+1}-\tau_{1}^{u}}\left|u(\nu)-v^{h}(\nu)\right|^{2} d \nu .
$$

Note that $\tau=l \tau_{1}^{u}+\gamma, \gamma \geq 0$. Therefore, $t_{j+1}-\tau_{1}^{u}=t_{j}, t_{j-l-1} \leq t_{j}-\tau \leq t_{j-l}$. In this case, for $t \in \Delta^{(j)}$ we have

$$
\varepsilon_{j}(t) \leq \varepsilon_{j}\left(t_{j}\right)+K_{7}^{(j)}(h+\delta)+K_{9}^{(j)} \sum_{k=j-l}^{j} \nu^{(k)} .
$$

Here, constants $K_{k}^{(j)}, k \in[0: 9]$ are written explicitly. Thus, one can assume that $c_{j}^{(1)}=K_{7}^{(j)}$ and $c_{j}^{(2)}=K_{9}^{(j)}$. The lemma is proved.

Lemma 4. Let $\delta \leq h$ and values $\alpha_{j}$ be given by (12). Then the inequalities

$$
\begin{gathered}
\nu^{(j)} \leq c_{j} g_{j}(h), \\
b_{j} \leq c_{j}^{(0)} g_{j}(h)
\end{gathered}
$$

are valid. 
Proof. For simplicity, set $t_{j_{*}+1}=\vartheta$. By virtue of lemma 3, we have for $t \in \Delta^{(j)}$

$$
|x(t)-w(t)| \leq\left(\varepsilon_{j}(t)+\alpha_{j} \int_{t_{j}}^{t}\left\{\left|v^{h}(\nu)\right|^{2}+|u(\nu)|^{2}\right\} d \nu\right)^{1 / 2} \leq\left(b_{j}+\alpha_{j} \rho_{A}\right)^{1 / 2},
$$

where $\rho_{A}=2 \tau_{*} d^{2}(P)$ and $d(P)=\sup \{|u|: u \in P\}$. Taking into account the inclusion $t_{j} \in \Delta_{h}$, we conclude that for any $j \in\left[0: j_{*}\right]$, one can specify the number $i=i_{j}(h)$ such that $t_{j}=\tau_{i_{j}(h)}$. Introduce the notation $\varrho_{j} \equiv \mid f_{1}(\cdot)-$ $\left.F_{1}(\cdot)\right|_{L_{2}\left(\Delta^{(j)} ; R^{\left.n_{2}\right)}\right.} ^{2}$. In this case, by virtue of lemma 2, as well as of (41) and (16), we obtain

$$
\varrho_{j} \leq d_{j}^{(1)} \sum_{i=i_{j}(h)}^{i=i_{j+1}(h)-1} \int_{\tau_{i}}^{\tau_{i+1}}\left\{\delta^{2}+h^{2}+\gamma^{h}(\nu)+\gamma_{i}^{h}(\nu)+\left|\xi^{h}\left(\tau_{i}\right)-w\left(\tau_{i}\right)\right|^{2}\right\} d \nu,
$$

where

$\gamma^{h}(\nu)=\sum_{k=1}^{m}\left|u\left(\nu-\tau_{k}^{u}\right)-v^{h}\left(\nu-\tau_{k}^{u}\right)\right|^{2}, \quad \gamma_{i}^{h}(\nu)=\sum_{k=0}^{n}\left|x\left(\nu-\tau_{k}^{x}\right)-\xi^{h}\left(\tau_{i}-\tau_{k}^{x}\right)\right|^{2}$.

Note that

$$
\begin{gathered}
\int_{t_{j}}^{t_{j+1}} \gamma^{h}(\nu) d \nu \leq d_{j}^{(2)} \int_{t_{j-l-1}}^{t_{j}}\left|u(\nu)-v^{h}(\nu)\right|^{2} d \nu=d_{j}^{(2)} \sum_{k=j-l}^{j} \nu^{(k)}, \\
\int_{t_{j}}^{t_{j+1}} \gamma_{i}^{h}(\nu) d \nu \leq d_{j}^{(3)}\left(h^{2}+\delta^{2}\right) .
\end{gathered}
$$

In addition,

$$
\nu^{(k)}=0 \quad k \in[-l: 0] .
$$

Therefore, combining inequalities (22)-(24), we obtain the estimates

$$
\varrho_{j} \leq d_{j}^{(5)}\left\{h^{2}+\delta^{2}+\sum_{k=j-l}^{j} \nu^{(k)}+b_{j}+\alpha_{j}\right\}, \quad j \in\left[0: j_{*}\right]
$$

One can easily see that the following estimates also hold:

$$
\left|f_{2}(\cdot)-F_{2}(\cdot)\right|_{L_{2}\left(\Delta^{(j)} ; R^{\left.n_{2} \times n_{1}\right)}\right.}^{2} \leq d_{j}^{(5)}\left(h^{2}+\delta^{2}\right), \quad j \in\left[0: j_{*}\right] .
$$

Here $d_{j}^{(1)}-d_{j}^{(5)}$ are some constants, which can be explicitly written. By lemma 3 (22), and (25), for $\delta \leq h$, we have the inequalities

$$
\varepsilon_{0}(t) \leq b_{0} \leq c_{0}^{*} h, \quad t \in \Delta^{(0)},
$$




$$
\left|x\left(t_{1}\right)-w\left(t_{1}\right)\right|^{2} \leq \rho_{A} \alpha_{0}+c_{0}^{*} h \leq c_{*} h^{2 / 3} .
$$

Taking into account (25)-(28) , for $h \in(0,1)$, we obtain

$\varrho_{0} \leq d_{0}^{(1)}\left\{h^{2}+\delta^{2}+b_{0}+h^{2 / 3}\right\} \leq d_{0}^{*} h^{2 / 3}, \quad\left|f_{2}(\cdot)-F_{2}(\cdot)\right|_{L_{2}\left(\Delta^{(0)} ; R^{\left.n_{2} \times n_{1}\right)}\right.}^{2} \leq c_{j}^{(*)} h^{2}$.

By virtue of condition 1, one can use lemma11. Set $p=x, q=w, u_{1}=u, u_{2}=v^{h}$, $f_{1}(t)=f_{1}\left(t, u_{t}(s), x_{t}(s)\right), f_{2}(t)=f_{2}\left(t, x_{t}(s)\right), F_{1}(t)=f_{1}\left(\tau_{i}, v_{\tau_{i}}^{h}(s), \xi_{\tau_{i}}^{h}(s)\right)+$ $2\left(\xi^{h}\left(\tau_{i}\right)-w\left(\tau_{i}\right)\right), F_{2}(t)=f_{2}\left(\tau_{i}, \xi_{\tau_{i}}^{h}(s)\right) \quad t \in\left[\tau_{i}, \tau_{i+1}\right)$. Then, assuming $a_{1}^{(0)}=$ $d_{0}^{*} h^{2 / 3}, a_{2}^{(0)}=c_{j}^{(*)} h^{2}, a_{3}^{(0)}=c_{0}^{*} h, a_{4}^{(0)}=c_{*} h^{2 / 3}, \tilde{\alpha}_{0}=\alpha_{0}=c h^{2 / 3}$, we have

$$
\nu^{(1)}=\left|u(\cdot)-v^{h}(\cdot)\right|_{L_{2}\left(\Delta^{(0)} ; R^{n_{1}}\right)}^{2} \leq \tilde{c}_{1} h^{1 / 3}=c_{1} g_{1}(h) .
$$

It means that inequality (20) holds for $j=1$. Further, by using (29) and (30), we deduce that

$$
b_{1}=\left|x\left(t_{1}\right)-w\left(t_{1}\right)\right|^{2}+c_{1}^{(1)}(h+\delta)+c_{1}^{(2)} \sum_{k=1-l}^{1} \nu^{(k)} \leq \tilde{c}_{1}^{(0)} h^{1 / 3}=c_{1}^{(0)} g_{1}(h) .
$$

Inequality (21) for $j=1$ is also verified. It follows from (22) that

$$
\left|x\left(t_{j}\right)-w\left(t_{j}\right)\right|^{2} \leq b_{j-1}+\rho_{A} \alpha_{j-1}, \quad j \in\left[1: j_{*}-1\right] .
$$

Consequently, in view of relations (31), as well as of the rule for definition $b_{j}$, we have the inequality

$$
b_{j} \leq b_{j-1}+d_{j}\left(h+\alpha_{j-1}+\sum_{k=j-l}^{j} \nu^{(k)}\right), \quad d_{j}=\text { const } \in(0,+\infty) .
$$

Setting $a_{1}^{(j)}=d_{j}^{(4)}\left\{h^{2}+\delta^{2}+\sum_{k=j-l}^{j} \nu^{(k)}+a_{3}^{(j)}+\alpha_{j}\right\}, a_{3}^{(j)}=b_{j}, a_{2}^{(j)}=d_{j}^{(5)}\left(h^{2}+\right.$ $\left.\delta^{2}\right), a_{4}^{(j)}=b_{j-1}+\rho_{A} \alpha_{j-1}, j \in\left[1: j_{*}\right]$ for $j \geq 1$ in lemma 1 and taking into account inequalities (32), we obtain

$\nu^{(j+1)} \leq c^{(j)}\left\{h^{1 / 2}+\left(\sum_{k=j-l}^{j} \nu^{(k)}\right)^{1 / 2}+b_{j-1}^{1 / 2}+\alpha_{j-1}^{1 / 2}+\alpha_{j}^{1 / 2}\right\}+b_{j} \alpha_{j}^{-1}, j \in\left[1: j_{*}\right]$.

Here, we used lemma 3 and inequalities (27), (28), and (31)) for choosing values $a_{i}^{(j)}$. Now, to proof inequalities (20) and (21), one can use the proof by induction. The lemma is proved.

\section{Example}

The algorithm was tested by a model example. The following system

$$
\begin{aligned}
& \dot{x}_{1}(t)=2 x_{1}(t-1)+u(t) \\
& \dot{x}_{2}(t)=x_{2}(t-1)+x_{1}(t)+u(t-1), \quad t \in T=[0,2],
\end{aligned}
$$


with initial conditions $x_{0}(s)=y_{0}(s)=1, u(s)=0$ for $s \in[-1,0]$ and control $u(t)=t$ was considered. The solution $x(t)=\left\{x_{1}(t), x_{2}(t)\right\}$ of system (33) was calculated analytically. During the experiment, we assumed that $\xi^{h}\left(\tau_{i}\right)=$ $x_{1}\left(\tau_{i}\right)+h$. As a model, we took the system (9), which has the form

$$
\begin{aligned}
& \dot{w}^{(0)}(t)=2 \xi_{1}^{h}\left(\tau_{i}-1\right)+v_{i}^{h}+2\left(\xi_{1}^{h}\left(\tau_{i}\right)-w^{(0)}\left(\tau_{i}\right)\right) \quad \text { for } \quad t \in\left[\tau_{i}, \tau_{i+1}\right) \\
& \dot{w}^{(1)}(t)=\xi_{2}^{h}\left(\tau_{i}-1\right)+\xi_{1}^{h}\left(\tau_{i}\right)+v^{h}\left(\tau_{i}-1\right)+2\left(\xi_{2}^{h}\left(\tau_{i}\right)-w^{(1)}\left(\tau_{i}\right)\right),
\end{aligned}
$$

with the initial condition $w^{(0)}(s)=w^{(1)}(s)=1$, for $s \in[-1,0]$. Here $v^{h}\left(\tau_{i}\right)=v_{i}^{h}$ for $t \in\left[\tau_{i}, \tau_{i+1}\right), i \geq 0, v^{h}(s)=0$ for $s \in[-1,0)$. The controls $v_{i}^{h}$ in model (34) were calculated by the following formula (see (11))

$$
v_{i}^{h}=\arg \min \left\{2 l_{i} v+\alpha_{j}|v|^{2}:|v| \leq K\right\},
$$

where $l_{i}=w^{(0)}\left(\tau_{i}\right)-\xi_{1}^{h}\left(\tau_{i}\right)$.

In figures 1 and 2 the results of calculations are presented for the case when $\delta=10^{-4}, \alpha_{0}=C h^{2 / 3}, \alpha_{1}=C h^{2 / 9}, C=0.2, K=10$. Fig. 1 corresponds to the case when $h=0.001$, fig. $2-h=0.02$. In these figures the solid (dashed) lines represent the model control $v^{h}(\cdot)$ (the real control $\left.u(\cdot)\right)$. The equations were solved by the Euler method with step $\delta$.

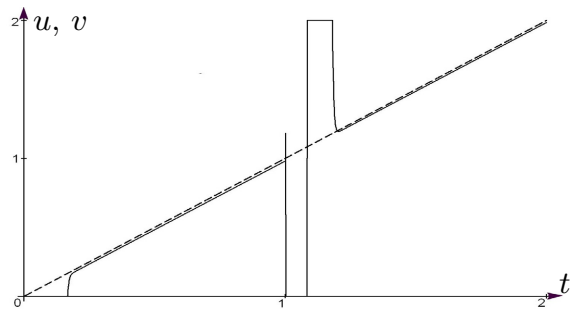

Fig. 1.

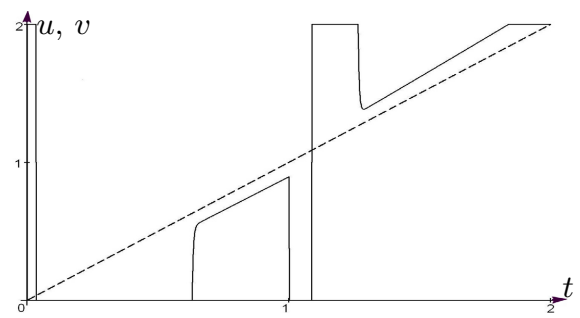

Fig. 2.

\section{References}

1. Osipov, Y.S., Kryazhimskii, A.V.: Inverse Problems for Ordinary Differential Equations: Dynamical Solutions. Gordon and Breach, London (1995)

2. Maksimov, V.I.: Dynamical Inverse Problems of Distributed Systems. VSP, UtrechtBoston (2002)

3. Maksimov, V., Pandolfi, L.: Dynamical reconstruction of inputs for constraction semigroup systems: the boudary input case. J. Optim. Theor. Appl. 103, 401-420 (1999)

4. Maksimov, V., Troltzsch, F.: Dynamical state and control reconstruction for a phase field model. Dynamics of Continuous, Discrete and Impulsive Systems. A: Mathematical Analysis 13(3-4), 419-444 (2006)

5. Osipov, Y.S., Kryazhimskii, A.V., Maksimov, V.I.: Methods of Dinamical Reconstruction of Inputs of Controlled Systems. Ekaterinburg (2011) (in Russian)

6. Maksimov, V.: Lyapunov function method in input reconstruction problems of systems with aftereffect. J. Math. Sci. 140(6), 832-849 (2007) 